\title{
A critical analysis of minor cardiovascular criteria in the diagnostic evaluation of patients with Marfan syndrome
}

Julie De Backer, $M D^{1,2}$, Bart Loeys, $M D$, PhD ${ }^{1}$, Dan Devos, $M D^{3}$, Harry Dietz, $M D, P h D^{4}$, Johan De Sutter, $\mathrm{MD}, \mathrm{PhD}^{2}$, and Anne De Paepe, $\mathrm{MD}, \mathrm{PhD}^{1}$

\begin{abstract}
Purpose: The prevalence of most minor cardiovascular manifestations in Marfan syndrome (MFS) is unknown. We assessed the prevalence of minor cardiovascular manifestations in MFS to evaluate their usefulness in a diagnostic setting. Methods: Seventy-seven patients with MFS (aged 4 months to 55 years) underwent echocardiography to assess the presence of mitral valve prolapse and the diameter of the main pulmonary artery. A subset of 29 adult patients with MFS also underwent magnetic resonance imaging evaluation of the diameters of the thoracoabdominal aorta. Results: Mitral valve prolapse was encountered in $66 \%$ of patients with MFS, with an equal distribution of classic and nonclassic mitral valve prolapse. The main pulmonary artery diameter was significantly larger in patients with MFS at all ages when compared with controls. In the adult group ( $\geq 14$ years), we were able to provide a cutoff value of $23 \mathrm{~mm}$ to define pulmonary artery dilatation. The descending aorta was enlarged, but with substantial overlap with controls, thus precluding the use of a cutoff value. Conclusions: Mitral valve prolapse and main pulmonary artery dilatation are common findings in MFS patients at all ages and are easy to assess with echocardiography. Cutoff values to define dilatation of the descending aorta are hard to define, making them of limited value in the diagnostic evaluation. We recommend echocardiographic evaluation of mitral valve proplase and main pulmonary artery diameter in patients referred for cardiovascular diagnostic assessment for MFS. Genet Med 2006:8(7):401-408.
\end{abstract}

Key Words: Marfan syndrome, diagnostic criteria, cardiovascular

Marfan syndrome (MFS) is an autosomal dominant connective tissue disorder characterized by a combination of clinical manifestations in different organ systems, including the skeletal, cardiovascular, ocular, and central nervous system. The diagnosis is mainly based on clinical characteristics and requires the identification of major and minor criteria, as summarized in the Gent nosology. ${ }^{1}$ Major criteria are specific for MFS; minor criteria are much less specific and occur frequently in the general population. The diagnosis in an index patient is confirmed by the presence of major criteria in at least two different organ systems in association with involvement of a third organ system. In patients with a first-degree relative who independently meets these diagnostic criteria, the presence of a major criterion in one organ system together with the involvement of a second organ system confirms the diagnosis of MFS.

\footnotetext{
From the ${ }^{I}$ Centre for Medical Genetics, ${ }^{2}$ Department of Cardiology, and ${ }^{3}$ Department of Medical Imaging, University Hospital Ghent, Ghent, Belgium; and ${ }^{4} \mathrm{McKusick-Nathans} \mathrm{Institute} \mathrm{for} \mathrm{Ge-}$ netic Medicine, Johns Hopkins University School of Medicine, Baltimore, Maryland.

Julie De Backer, MD, Centre for Medical Genetics University Hospital Ghent, Belgium De Pintelaan 1859000 Ghent, Belgium.

Submitted for publication January 18, 2006.

Accepted for publication March 31, 2006.

DOI: 10.1097/01.gim.0000223550.41849.e3
}

The importance of a correct interpretation of minor manifestations is twofold.

First, adequate interpretation of minor manifestations may be important in excluding the diagnosis of MFS. In cases with a marfanoid habitus with myopia, for example, the absence of any cardiovascular manifestation may be reassuring.

Second, it may be essential for the confirmation of the diagnosis in patients in whom involvement of a third organ system is required, for example, in children with lens luxation and major skeletal manifestations, but without striae.

With regard to the cardiovascular system, mitral valve prolapse (MVP) is the only manifestation for which the investigational method and definition are well delineated. Whereas MVP was initially diagnosed with M-mode techniques, the current guidelines recommend two-dimensional echocardiography.,3 MVP is further subdivided into classic and nonclassic MVP, according to whether valve thickening is present or not. Nonclassic MVP is often referred to as "mitral valve bulging" in cardiovascular literature. To the best of our knowledge, the prevalence of these two different forms of MVP has not been assessed in patients with MFS.

The true prevalence of the other minor cardiovascular criteria in MFS is largely unknown. Normal values for main pulmonary artery (MPA) diameters are only available for children, ${ }^{4}$ and the prevalence of MPA dilatation has only been assessed with magnetic resonance imaging (MRI) in patients with MFS. ${ }^{5} \mathrm{~A}$ few 
reports on normal values for the diameters of the descending thoracic or abdominal aorta are available, ${ }^{6,7}$ but these have not yet been evaluated systematically in patients with MFS.

The objectives of this study are as follows:

1. Evaluate the prevalence of minor cardiovascular manifestations in a large group of patients with MFS: MVP, dilatation of the MPA in a patient aged less than 40 years, dilatation or dissection of the descending thoracic or abdominal aorta in a patient aged less than 50 years, and calcification of the mitral annulus in a patient aged less than 40 years.

2. Propose recommendations for the assessment of minor cardiovascular criteria in MFS, based on the findings in this study.

\section{MATERIALS AND METHODS}

A total of 77 subjects with a mean age of 25.0 years (range 4 months to 55 years) who fulfilled the Gent criteria for MFS were studied. We defined two groups: children (aged $<14$ years; $\mathrm{N}=24$ ) and adults (aged $\geq 14$ years; $\mathrm{N}=53$ ). Five patients (four men, one woman; all aged $\geq 14$ years) had previously undergone aortic root surgery; one patient (28-yearold female) had previously undergone mitral valve replacement for severe mitral valve regurgitation based on prolapse.

Patients were compared with 77 age- and sex-matched control subjects, recruited among colleagues and family members of the researchers. None of the control subjects had known cardiovascular disease.

A subgroup of 29 adult patients with MFS and 31 control subjects also underwent MRI imaging of the aorta.

\section{Echocardiography}

Transthoracic echocardiography was performed on a VIVID 7 Vingmed-General Electric scanner (GE Vingmed Ultrasound, Horten, Norway). MVP was evaluated from the parasternal longaxis view and the apical four-chamber view. Classic MVP was defined as leaflet displacement exceeding $2 \mathrm{~mm}$ and leaflet thickness of at least $5 \mathrm{~mm}$; nonclassic MVP was defined as leaflet displacement exceeding $2 \mathrm{~mm}$ and leaflet thickness less than $5 \mathrm{~mm}$, according to Freed et al. ${ }^{2}$

The MPA diameter was measured a few millimeters distally from the valve insertion at the broadest portion of the pulmonary artery, visualized from the parasternal short-axis window (Fig. 1). Images were stored in digital format on a remote hard disk. Data were reviewed offline by a sonographer blinded to the diagnosis of the subject with a commercially available imageprocessing program (Echopac 6.3, GE Vingmed Ultrasound).

To test interobserver variability of the measurement, the images of a randomly selected subset of 10 patients with MFS and 10 controls were evaluated by an independent sonographer. For the evaluation of intraobserver variability, the acquired images of 31 patients with MFS were analyzed twice by the same sonographer. Intra- and interobserver variability were determined by the coefficients of variance by comparing

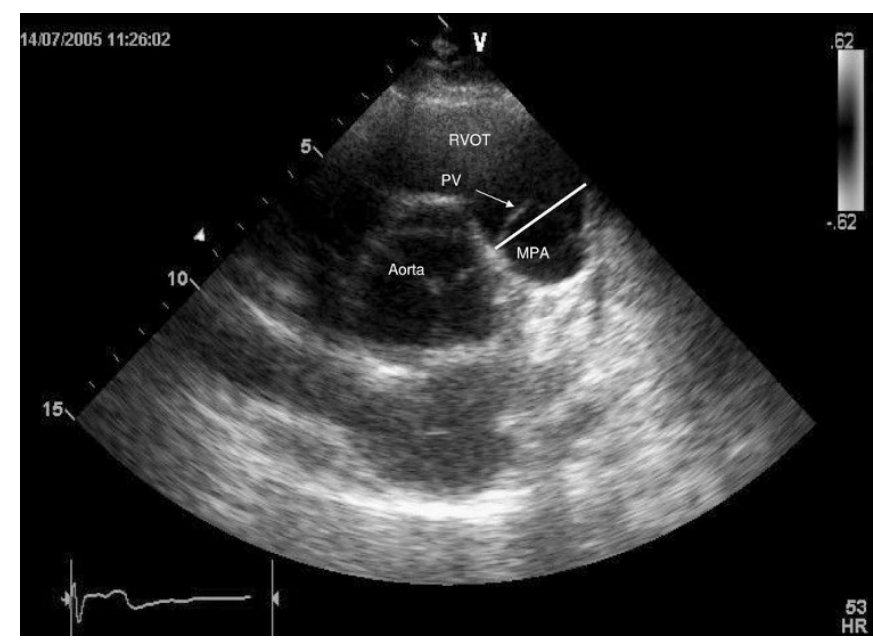

Fig. 1. Echocardiographic image of the MPA measurement. RVOT, right ventricular outflow tract; PV, pulmonary valve; MPA, main pulmonary artery.

the standard deviation (SD) of the test differences as a percentage of the average in both series.

\section{Magnetic resonance imaging}

MRI was performed on a 1.5T MR system (Magnetom Symphony, Siemens, Erlangen, Germany). Aortic diameter was assessed at the level of the ascending aorta (at the level of the pulmonary artery), descending thoracic aorta (at the level of the pulmonary artery), diaphragm, and abdomen (distally from the major abdominal branch vessels, just proximal to the bifurcation). A high temporal resolution (25 $\mathrm{msec})$ balanced fast gradient (TrueFISP) cine sequence was used, positioned in an optimal transverse plane of the aorta. On these images the largest (systolic) diameter was measured. Aortic aneurysms and dissections were evaluated with magnetic resonance angiography.

Body surface area (BSA), was calculated according to the DuBois and DuBois formula: ${ }^{8}$

$$
\mathrm{BSA}=0.20247 \times(\mathrm{H})^{0.725} \times(\mathrm{W})^{0.425}
$$

with $\mathrm{W}=$ weight in kilograms; $\mathrm{H}=$ height in meters.

\section{Statistical analysis}

Baseline characteristics were compared with nonpaired $t$ test for continuous variables and with chi-square tests for categoric data. Multivariate analysis was performed to assess the influence of covariates.

For patients and controls, mean diameters are given with their respective SD. The distribution of the diameters was normal. Student $t$ test was used to compare the two groups. A $P$ value of .05 or less was used to define statistical significance. SPSS version 11.0.1 (SPSS Inc., Chicago, IL) was used for the statistical analysis.

This study was approved by the local ethics committees (Ghent University Hospital; Johns Hopkins Institute). 


\section{RESULTS}

\section{Baseline characteristics}

Baseline characteristics in adults are presented in Table 1.

Age, sex, BSA, and BMI were comparable in both groups. As expected, patients with MFS were taller than controls $(P<$ .0001 ), and the diameter of the proximal aorta measured at the level of the sinus Valsalva was larger $(P<.0001)$. The subgroup of patients with MFS who also underwent MRI was not significantly different from the other patients with MFS with respect to age, sex, BSA, and BMI.

Children were aged 4 months to 14 years; 50\% were boys. The mean $z$ score of the aortic sinus was 4.56 (SD 3.1).

\section{Mitral valve prolapse and calcification of the mitral valve annulus}

In total, $66 \%$ of patients with MFS had some form of MVP. Classic MVP was present in $35.1 \%$ of patients with MFS; nonclassic MVP was present in 31.2\%. Mitral valve regurgitation was absent in all but two patients: One had moderate mitral valve regurgitation, and one (28-year-old female) had previously undergone mitral valve replacement (mechanical valve) for severe regurgitation because of underlying MVP. One control subject had nonclassic MVP without mitral valve regurgitation. Within the group of patients with MFS, MVP was not related to sex, length, or weight. Patients with nonclassic MVP were older than those without or with classic MVP (33.2 \pm 16.6 years vs. $25.0 \pm$ 11.5 years and $17.6 \pm 13.7$ years, respectively, $P=.001)$.

MVP was more common in children with MFS than in adults (79\% vs. $60 \% ; P=.003)$.

In this patient population, nobody demonstrated significant calcification of the mitral valve annulus on echocardiography.

\section{Dilatation of the main pulmonary artery}

Measurement of the MPA diameter was possible in all but three adult patients with MFS and in all but two control patients.

The MPA diameter was significantly larger in patients with MFS compared with controls: $25.1 \pm 4.7 \mathrm{~mm}$ versus $18.9 \pm 3.7$ $\mathrm{mm}(P<.001)$. In univariate analysis including age, BSA, sex, and the presence of MFS, BSA and the presence of MFS ap- peared as independent predictors of the MPA diameter. After multivariate analysis, the presence of MFS and BSA remained as the only independent predictors. The relationship between age and BSA and the MPA diameter for the different age categories ( $>14$ years and $\leq 14$ years) is illustrated in Figure $1 \mathrm{~A}$ to D. It can be appreciated from this that the MPA is dilated from early in life. The strong relationship between age and BSA and the MPA diameter in the younger age group is illustrated in Figure 1A and B, whereas this relationship becomes less pronounced in adulthood.

Subdividing the patients in the two age categories $(>40$ years or $<40$ years), as proposed in the current diagnostic criteria, does not alter the global finding of increased diameter in the adult patients with MFS (Fig. 2).

We found a significant correlation $\left(\mathrm{r}^{2}=0.54\right)$ between the diameter of the proximal aorta and the pulmonary artery annulus diameter (Fig. 3), although it is noteworthy that some patients with MFS with a normal diameter of the proximal aorta had significant dilatation of the MPA.

A receiver operating characteristic curve to evaluate the value of measurement of the MPA root in the prediction of the disease was constructed. In a receiver operating characteristic curve, true positives are plotted against the false-positive rate for the different cutpoints of a diagnostic test. The area under the curve is a measure of test accuracy (with an area of 1 representing a perfect test and an area of 0.5 representing a worthless test). When applied on the entire study population, the area under the curve was 0.85 .

Limiting this analysis to the age group 14 years and older increased this value to 0.94 (Fig. 4), indicating that measurement of the MPA diameter can adequately discriminate patients with MFS from controls in this age group. On the basis of this curve, cutoff values with a certain sensitivity and specificity are calculated. We propose a cutoff value of $23 \mathrm{~mm}$ for MPA diameter in subjects 14 years and older, corresponding to a sensitivity of $85 \%$ and a specificity of $94.2 \%$.

In children, a uniform cutoff value is not applicable because of the strong relationship with BSA in this age group. We rec-

Table 1

Baseline characteristics for adult patients with Marfan syndrome and controls

\begin{tabular}{|c|c|c|c|c|}
\hline & $\operatorname{Marfan}(\mathrm{N}=53)$ & Marfan MRI subgroup $(\mathrm{N}=29)$ & Controls $(\mathrm{N}=53)$ & $P$ value $^{a}$ \\
\hline Age $(y)$ & $33.0 \pm 10.9$ & $31.9 \pm 10.4$ & $37.3 \pm 11.9$ & .06 \\
\hline Male sex (\%) & 47 & 48 & 56 & .38 \\
\hline Length $(\mathrm{cm})$ & $184.3 \pm 9.0$ & $184.3 \pm 9.7$ & $172.4 \pm 9.7$ & $<.0001$ \\
\hline Weight (kg) & $73.7 \pm 15.0$ & $77.7 \pm 13.7$ & $70.7 \pm 13.8$ & .27 \\
\hline $\operatorname{BSA}\left(\mathrm{m}^{2}\right)$ & $1.92 \pm 0.3$ & $2.0 \pm 0.2$ & $1.83 \pm 0.2$ & .191 \\
\hline BMI $\left(\mathrm{kg} / \mathrm{m}^{2}\right)$ & $22.2 \pm 4.1$ & $23.0 \pm 4.2$ & $23.5 \pm 3.8$ & .018 \\
\hline $\begin{array}{l}\text { Aortic diameter at the level of } \\
\text { the sinus Valsalva }(\mathrm{mm})^{b}\end{array}$ & $41.6 \pm 1.0$ & $39.8 \pm 0.8$ & $29.7 \pm 0.3$ & $<.0001$ \\
\hline
\end{tabular}

Continuous values given as mean \pm standard deviation.

${ }^{a}$ Between patients with Marfan syndrome (MFS) and controls.

${ }^{b}$ Only assessed in patients without previous aortic root surgery.

MRI, magnetic resonance imaging; BSA, body surface area; BMI, body mass index. 

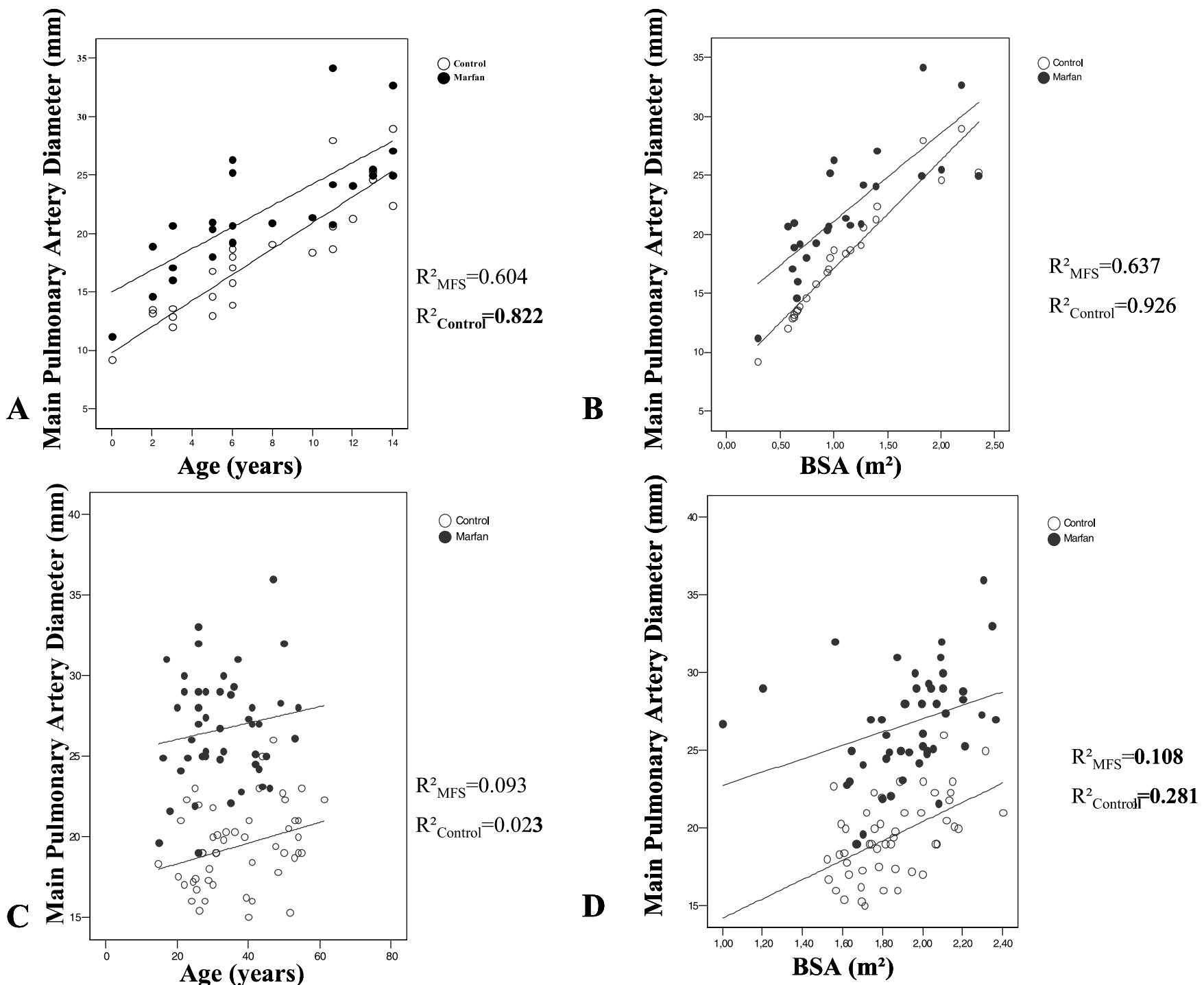


years).

ommend the use of $z$ scores in this age group; normal values were published by Snider et al. ${ }^{9}$ They provide a regression equation for calculating the MPA diameter based on the BSA:

MPA diameter (in centimeters) $=0.0946+1.544 \mathrm{BSA}^{1} \frac{1}{2}$, with an SD of 0.32 . From this, the $z$ score for an individual patient can be calculated as

$$
\mathrm{Z}=\mathrm{X}-\mathrm{M} / \mathrm{SD}
$$

with $X=$ the obtained value for a specific subject, $M=$ the mean expected for that subject, and SD the standard deviation of the expected mean value, being 0.32 in this case.

The major benefit of the proposed cutoff value is its excellent negative predictive value. Nevertheless, it may occasionally be helpful for confirmation of the diagnosis. We identified cardiovascular involvement in five adult patients with MFS without dilatation of the proximal aorta $(z$ score $<2)$ and without MVP. The clinical characteristics of these patients are given in Table 2. All of these subjects were family members of pa- tients who fulfilled the diagnostic criteria; they were all identified on the basis of the presence of a FBN1 mutation. In many centers, however, mutation screening is not performed on a routine basis, and in these instances, correct assessment of clinical manifestations may be crucial.

Interobserver and intraobserver variability of the echocardiographic measurements were good with coefficients of variance of $3.5 \%$ for intraobserver variability and $6.5 \%$ for interobserver variability.

\section{Dilatation and dissection of the descending thoracic or abdominal aorta}

When the mean values for the aortic diameters at different levels (excluding one outlier with an aneurysm described further in the text) were compared, significant differences were found between patients with MFS and control subjects (Table 3). However, absolute differences were small, and there was 


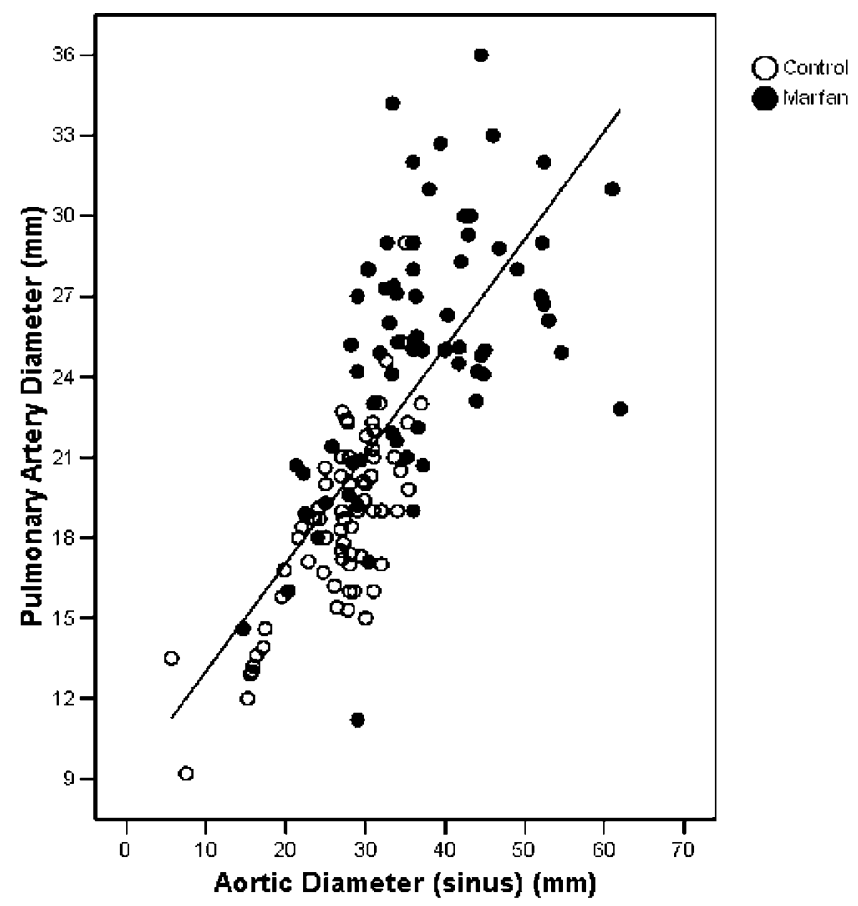

Fig. 3. Correlation between the diameter of the proximal aorta and the main pulmonary artery diameter.

substantial overlap between patients and controls, which compromised our attempt to set up reference values (Fig. 5).

We calculated $z$ scores based on our findings in control subjects $(z$ score $=[$ obtained value - mean value $] / S D)$. At the level of the descending thoracic aorta, six patients $(21 \%)$ had a $z$ score of 2 or

\section{ROC Curve}

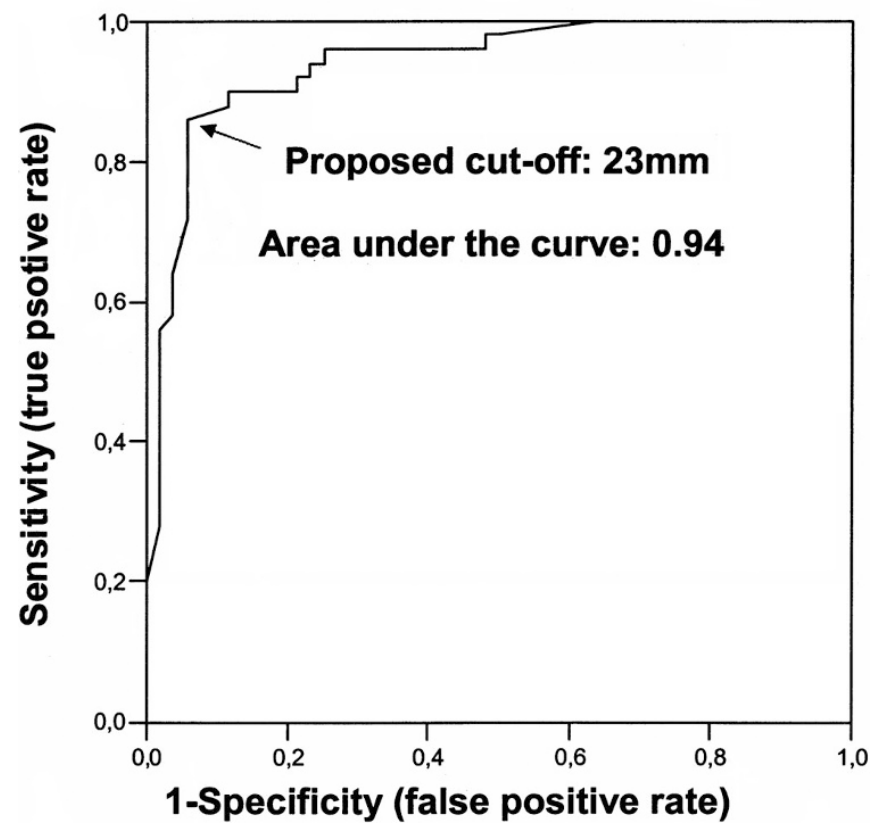

Fig. 4. Receiver operating characteristic curve assessing the accuracy of pulmonary artery root diameter measurement in the diagnosis of adult patients with Marfan syndrome. greater, four of whom had previous aortic root surgery; at the level of the diaphragm, three patients (10\%) had a $z$ score of 2 or greater, two of whom underwent previous aortic root surgery; at the level of the abdominal aorta, two patients (7\%) had a $z$ score of 2 or greater, one of whom underwent previous aortic root surgery. Except for the patient with an aneurysm of the descending aorta, the $z$ scores were all less than 3 .

In patients with MFS and in controls, the diameters at the different levels of the descending aorta correlated, but in patients with MFS, there was no significant correlation with the diameter at the aortic sinus or the pulmonary artery.

We detected an asymptomatic type $\mathrm{B}$ dissection in one patient (29-year-old woman) with a significant dilatation of the descending aorta (diameter of 5.7, 5.6, and $3.5 \mathrm{~cm}$ at the level of the descending thoracic aorta, diaphragm, and abdominal aorta, respectively). Of interest is that this particular patient had a normal diameter of the proximal aorta $(3.6 \mathrm{~cm}$ at the level of the sinuses of Valsalva, $z$ score 1.4). One control subject (52-year-old man) also had slightly elevated diameters of the descending thoracic aorta (diameter of 2.6, 3.4, and $2.6 \mathrm{~cm}$ at the level of the descending thoracic aorta, diaphragm, and abdominal aorta, respectively). This patient had no cardiovascular risk profile, and family history for abdominal aortic aneurysm was negative.

\section{DISCUSSION}

Cardiovascular manifestations in MFS have been defined in the Gent nosology in 1996. ${ }^{1}$ At that time, however, clear guidelines for the assessment of these manifestations were limited to measurement of the diameter of the proximal aorta, as defined by Roman et al., ${ }^{10}$ and to the standardized imaging techniques for the assessment of ascending aortic dissection. Since then, standardized two-dimensional echocardiographic methods have been published, and large-scale trials have reported on the prevalence of classic and nonclassic MVP in the general population, ${ }^{2,11}$ but the prevalence of these two forms of MVP in MFS according to these guidelines has not yet been assessed. The absence of validated techniques and reference values for the other minor criteria, namely, dilatation or dissection of the descending thoracic aorta, dilatation of the MPA, and calcification of the mitral valve annulus, have hampered the use of these criteria in the diagnosis of MFS.

In this study we evaluated the prevalence of these minor cardiovascular criteria in patients with MFS and attempted to formulate practical guidelines for cardiovascular assessment in patients referred for diagnostic evaluation of MFS.

The prevalence of MVP in patients with MFS was $66.2 \%$, with nearly equal proportions of classic and nonclassic MVP. These figures clearly confirm a higher prevalence of MVP in patients with MFS when compared with the general population $(1.3 \%$ for the classic form and $1.1 \%$ for the nonclassic form), ${ }^{2}$ as already stated by other authors. ${ }^{12,13}$ Nonclassic MVP, in cardiovascular literature often referred to as "mitral valve bulging," should equally be considered in the diagnostic evaluation. 
Table 2

Clinical characteristics of the five patients with pulmonary artery dilatation as the only cardiovascular manifestation

\begin{tabular}{|c|c|c|c|c|c|}
\hline $\begin{array}{l}\text { Patient } \\
\text { (age/sex) }\end{array}$ & Skeletal system & Eyes & Skin & Dural ectasia & Family/genetic \\
\hline $1(27 / \mathrm{F})$ & $\begin{array}{l}\text { Pectus carinatum, severe scoliosis, arachnodactyly, } \\
\text { increased arm span, flat feet, joint } \\
\text { hypermobility, typical facial appearance }\end{array}$ & Myopia & Striae & Present & $\begin{array}{l}\text { Brother affected } \\
\text { FBN1: c. } 7828 \mathrm{G}>\mathrm{A}\end{array}$ \\
\hline $2(26 / \mathrm{F})$ & $\begin{array}{l}\text { Pectus excavatum, scoliosis, increased arm span, } \\
\text { flat feet, facial appearance, hypermobility }\end{array}$ & Ectopia lentis & No striae & Not assessed & $\begin{array}{l}\text { Brother affected } \\
\text { FBN1: c. } 1463 \mathrm{G}>\mathrm{T}\end{array}$ \\
\hline $3(28 / \mathrm{F})$ & $\begin{array}{l}\text { Arachnodactyly, increased arm span, reduced } \\
\text { elbow extension, flat feet, facial appearance }\end{array}$ & Retinal detachment & No striae & Absent & $\begin{array}{l}\text { Mother affected } \\
\text { FBN1: IVS8-1G >C }\end{array}$ \\
\hline $4(20 / \mathrm{F})$ & $\begin{array}{l}\text { Arachnodactyly, increased arm span, decreased } \\
\text { elbow extension, flat feet, facial appearance, } \\
\text { joint hypermobility }\end{array}$ & Myopia & No striae & Not assessed & $\begin{array}{l}\text { Father affected } \\
\text { FBN1: c. } 408 \mathrm{C}>\mathrm{A}\end{array}$ \\
\hline $5(21 / \mathrm{F})$ & $\begin{array}{l}\text { Arachnodactyly, flat feet, increased arm span, } \\
\text { reduced elbow extension, joint hypermobility }\end{array}$ & Not affected & No striae & Not assessed & $\begin{array}{l}\text { Father and brother affected } \\
\text { FBN1: IVS8-1G }>C\end{array}$ \\
\hline
\end{tabular}

In accordance with the figures from previous publications, we found a relatively higher prevalence of MVP in children with MFS when compared with adults. This may be because MVP is often the presenting cardiovascular sign of MFS in children, whereas in adults other manifestations might have led to the diagnosis.

Calcification of the mitral valve annulus may be a specific manifestation in patients with MFS, but this parameter is difficult to quantify with echocardiography. Although it is not possible to exclude a higher prevalence of mitral annular calcification if more sensitive screening practices such as computed tomography were used, it seems unlikely that the yield would justify the added inconvenience and expense on patients.

Guidelines regarding measurement and normal values for MPA dilatation were lacking at the time of the publication of the diagnostic criteria.

Measurement of the MPA diameter may be useful in ruling out cardiovascular involvement suggestive of MFS.

The prevalence of MPA dilatation in patients with MFS was recently studied by Nollen et al. ${ }^{5}$ with the use of MRI.

Complications arising from pulmonary artery dilatation are much milder, if any, compared with those resulting from progressive aortic dilatation. This is mainly attributable to the lower pressure in the pulmonary circulation. Pulmonary artery dissection is rare.

The excellent negative predictive value of the proposed cutoff value of $23 \mathrm{~mm}$ indicates that cardiovascular involvement can be ruled out with high certainty when measuring a diameter less than $23 \mathrm{~mm}$ in patients aged 14 years or more, given

Table 3

Thoracoabdominal aortic diameters at different levels (values in centimeters)

\begin{tabular}{lccr}
\hline & Marfan $(\mathrm{N}=27)$ & Control $(\mathrm{N}=25)$ & $P$ Value \\
\hline Descending thoracic & $24.1 \pm 2.7$ & $20.8 \pm 2.7$ & $<.001$ \\
Diaphragm & $21.7 \pm 2.7$ & $19.3 \pm 3.7$ & $<.001$ \\
Abdomen & $16.9 \pm 2.6$ & $14.9 \pm 3.1$ & .005 \\
\hline
\end{tabular}

that no aortic root dilatation or MVP is present. On the other hand, we identified five patients with definite MFS, in whom no other cardiovascular sign was present except for an MPA diameter exceeding $23 \mathrm{~mm}$.

Defining dilatation of the MPA in children is more problematic. Although the MPA is clearly dilated in the younger age group, one has to take both age and BSA into account. A uniform cutoff value in children cannot be defined. Instead, the use of $z$ scores should be recommended.

MPA diameter correlated to the diameter of the proximal aorta, indicating that they are the result of the same underlying pathophysiologic process. We encountered patients without significant aortic root dilatation who already had a dilated pulmonary artery, which enabled us to define involvement of the cardiovascular system in these patients.

Because dilatation of the MPA was observed in the entire age range under study here, it is preferable to abolish the age limit of 40 years, as proposed in the Ghent criteria.

Nollen and colleagues 5 measured the diameter of the MPA with MRI in patients with MFS. By using a cutoff value of 28 $\mathrm{mm}$ at the level of the MPA root, Nollen et al. report a prevalence of MPA dilatation of $74 \%$. There are several possible explanations for the difference in proposed cutoff values of 28 $\mathrm{mm}$ and our proposed cutoff value of $23 \mathrm{~mm}$. First, the site of measurement was different; whereas Nollen et al. measured the anterior/right diameter, we were limited by the echocardiographic window to measure the anterior/left window. Second, a significantly higher proportion of the patients studied by Nollen et al. had a history of aortic root surgery, indicating that this group of patients had more advanced involvement of the cardiovascular system with higher diameters of the pulmonary artery. Last, but not least, the applied imaging techniques in both studies are different and thus are not comparable.

One of the major advantages of MRI is that image acquisition is not limited by patient factors such as thorax deformities. This may be important in the case of patients with MFS, although we encountered only two patients in whom imaging of the MPA was inadequate with echocardiography. Another in- 



Fig. 5. Diameter of the descending aorta at three levels: descending thoracic, diaphragm, and abdominal.

teresting point is that both the aortic root and the MPA root are asymmetric, which has been nicely demonstrated using MRI. ${ }^{14,15}$ This asymmetry may also be part of the explanation for the difference in obtained values between our study and the MRI study by Nollen et al. ${ }^{15}$

In a diagnostic setting, however, echocardiography is preferable compared with MRI, in view of the higher costs and lower accessibility of MRI.

Data on dilatation of the descending aorta in patients with MFS are scarce. Patients with MFS presenting with thoracoabdominal aortic aneurysm/dissection are reported in a few case reports. ${ }^{16,17}$ Other reports on the descending aorta in patients with MFS are mainly limited to surgical data describing the occurrence of primary or secondary complications in the descending aorta necessitating surgical intervention. Finkbohner and colleagues ${ }^{18}$ report that $15 \%$ of their patients underwent initial surgery that involved portions of the descending aorta. Nollen and colleagues ${ }^{19}$ report an increased growth (defined as $>1 \mathrm{~mm} /$ year) in a small subset of patients $(6 \%$ in the descending thoracic aorta and 7\% in the abdominal aorta). Kawamoto and colleagues ${ }^{20}$ studied the progression of thoracoabdominal aortic diameters in patients with MFS after surgical repair and defined a subgroup of patients showing progressive dilatation of the distal aorta ( $>3 \mathrm{~mm} /$ year).

Our data demonstrate that as a group, patients with MFS have increased diameters at different levels of the thoracoabdominal aorta when compared with controls. There is, however, substantial overlap between the upper values of controls and the lower values of patients with MFS, precluding the use of a specific cutoff value. For this reason, we believe that measurement of descending aortic diameters is of little value in a diagnostic screening setting.

Our findings obtained from the calculation of the $z$ scores of the thoracoabdominal aorta show that increased $z$ scores $(\geq 2)$ occur only in a minority of patients and are more common in patients having previously undergone aortic root surgery. For this reason and in view of the results obtained in the studies mentioned earlier in postoperative patients with MFS, ${ }^{20,21} \mathrm{im}$ aging of the distal aorta should be performed on a regular basis in this subset of patients.

\section{Guidelines for cardiovascular assessment in patients referred for diagnostic cardiovascular evaluation for Marfan syndrome}

We propose the following flowchart for cardiovascular examination in adult patients referred for initial screening:

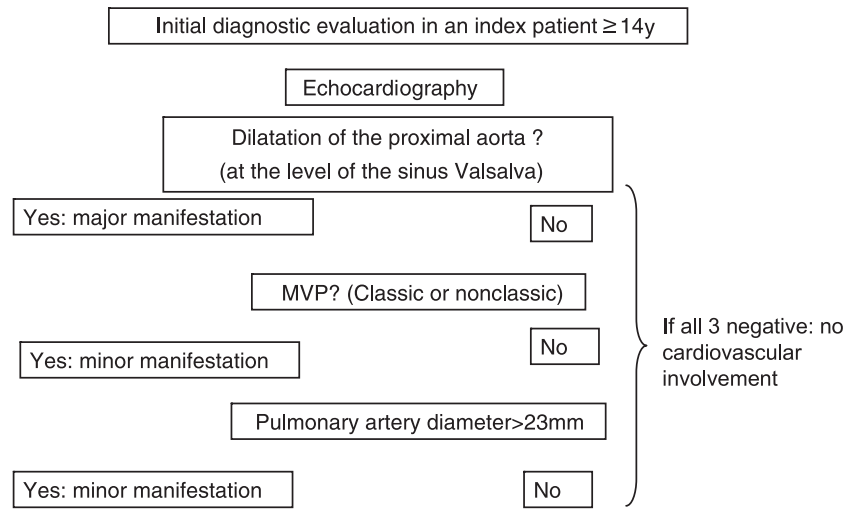

In children, the $z$ score for the MPA diameter should be calculated as described above. In the flowchart, "pulmonary artery diameter $>23 \mathrm{~mm}$ " should be replaced by "a $z$ score $>2$."

\section{Study limitations}

Matching with controls was done for age and sex, not for weight and height, but this was overcome by including weight and height as covariants in the statistical analysis. 
This study design was retrospective. Application of these guidelines in a prospective manner is necessary to confirm their validity.

\section{ACKNOWLEDGMENTS}

This study was supported by research grants from the Ghent University (BOF 011D4701) (J. De Backer) and the Fund for Scientific Research Belgium (FWO G029002) (A. De Paepe). Johan De Sutter and Bart Loeys are senior clinical investigators of the Fund for Scientific Research, Flanders (Belgium) (FWOVlaanderen).

\section{References}

1. De Paepe A, Devereux RB, Dietz HC, Hennekam RC, et al. Revised diagnostic criteria for the Marfan syndrome. Am J Med Genet 1996;62:417-426.

2. Freed LA, Levy D, Levine RA, Larson MG, et al. Prevalence and clinical outcome of mitral-valve prolapse. N Engl J Med 1999;341:1-7.

3. Levine R, Handschumacher M, Sanfilippo A, Hagege A, et al. Three-dimensional echocardiographic reconstruction of the mitral valve, with implications for the diagnosis of mitral valve prolapse. Circulation 1989;80:589-598.

4. Lorenz $\mathrm{CH}$. The range of normal values of cardiovascular structures in infants, children, and adolescents measured by magnetic resonance imaging. Pediatr Cardiol 2000;21:37-46.

5. Nollen GJ, van Schijndel KE, Timmermans J, Groenink M, et al. Pulmonary artery root dilatation in Marfan syndrome: quantitative assessment of an unknown criterion. Heart 2002;87:470-471.

6. Garcier JM, Petitcolin V, Filaire M, Mofid R, et al. Normal diameter of the thoracic aorta in adults: a magnetic resonance imaging study. Surg Radiol Anat 2003;25:322-329.

7. Hager A, Kaemmerer H, Rapp-Bernhardt U, Blucher S, et al. Diameters of the thoracic aorta throughout life as measured with helical computed tomography. J Thorac Cardiovasc Surg 2002;123:1060-1066.

8. Dubois DA. A formula to estimate the approximate surface area if height and weight be known. Arch Int Med 1916;17:863.
9. Snider AR, Enderlein MA, Teitel DF, Juster RP Two-dimensional echocardiographic determination of aortic and pulmonary artery sizes from infancy to adulthood in normal subjects. Am J Cardiol 1984;53:218-224.

10. Roman MJ, Devereux RB, Kramer-Fox R, O’Loughlin J Two-dimensional echocardiographic aortic root dimensions in normal children and adults. Am J Cardio 1989;64:507-512.

11. Freed LA, Benjamin EJ, Levy D, Larson MG, et al. Mitral valve prolapse in the general population: the benign nature of echocardiographic features in the Framingham Heart Study. J Am Coll Cardiol 2002;40:1298-1304.

12. van Karnebeek CDM, Naeff MSJ, Mulder BJM, Hennekam RCM, et al. Natural history of cardiovascular manifestations in Marfan syndrome. Arch Dis Child 2001; 84:129-137.

13. Yetman AT, Bornemeier RA, McCrindle BW. Long-term outcome in patients with Marfan syndrome: is aortic dissection the only cause of sudden death? J Am Coll Cardiol 2003;41:329-332.

14. Meijboom LJ, Groenink M, van der Wall EE, Romkes H, et al. Aortic root asymmetry in Marfan patients: evaluation by magnetic resonance imaging and comparison with standard echocardiography. Int J Card Imaging 2000;16:161-168.

15. Nollen GJ, van Schijndel KE, Timmermans J, Groenink M, et al. Magnetic resonance imaging of the main pulmonary artery: reliable assessment of dimensions in Marfan patients on a simple axial spin echo image. Int J Cardiovasc Imaging 2003;19:141147; discussion 9-50.

16. van Ooijen B. Marfan's syndrome and isolated aneurysm of the abdominal aorta. $\mathrm{Br}$ Heart J 1988;59:81-84.

17. Pruzinsky MS, Katz NM, Green CE, Satler LF. Isolated descending thoracic aortic aneurysm in Marfan's syndrome. Am J Cardiol 1988;61:1159-1160.

18. Finkbohner R, Johnston D, Crawford ES, Coselli J, et al. Marfan syndrome. Longterm survival and complications after aortic aneurysm repair. Circulation 1995;91: $728-733$.

19. Nollen GJ, Groenink M, Tijssen JG, Van Der Wall EE, et al. Aortic stiffness and diameter predict progressive aortic dilatation in patients with Marfan syndrome. Eur Heart J 2004;25:1146-1152.

20. Kawamoto S, Bluemke DA, Traill TA, Zerhouni EA Thoracoabdominal aorta in Marfan syndrome: MR imaging findings of progression of vasculopathy after surgical repair. Radiology 1997;203:727-732.

21. Finkbohner R, Johnston D, Crawford ES, Coselli J, et al. Marfan syndrome: longterm survival and complications after aortic aneurysm repair. Circulation 1995;91: $728-733$. 